\title{
IPR Management Models for Cultural Heritage on ECLAP Best Practice Network
}

\author{
Pierfrancesco Bellini, Paolo Nesi, Michela Paolucci \\ DISIT-DSI, Distributed Systems and Internet Technology Lab, \\ Dipartimento di Ingegneria dell'Informazione, Università degli Studi di Firenze, \\ Firenze, Italy \\ pbellini@dsi.unifi.it,paolo.nesi@unifi.it,paolucci@dsi.unifi.it
}

\begin{abstract}
Intellectual Property Rights (IPR) modelling allows to specify how a digital content can be used. Moreover, with the growing interest for the open source licensing of software artefacts the same 'open' policies have been applied also to digital content and thus open does not implies that one can do everything he like on the accessed content. The IPR management to allow the enforcement in play/access of specific conditions expressed in a license and their application during the content workflow, are complex and partially solved problems. In this paper, we describe the system adopted in the European Collected Library of Artistic Performance, ECLAP, to publish and distribute digital content taking into account the problems connected to the IPR of cultural heritage cross media content on Performing Arts domain. The paper reports IPR models and solutions adopted for IPR enforcement and the statistical analysis of about the effective usage of the solution proposed.
\end{abstract}

Keywords-Best Practice Network; Intellectual Property Rights management; licencing, Performing Arts digital contents.

\section{INTRODUCTION}

Intellectual Property Rights modelling, also called licensing, has often the aim to regulate the possible uses of the digital content (e.g., use on a specific device, use for a specific time period, use on a specific territory, use for educational purposes, etc.). In order to allow interoperability, standards have been developed for the formalization of licenses and access control policies like the MPEG-21 REL [1], ODRL [2], OASIS XAMCL [3]. For the definition of the actions, possible rights ontologies or vocabularies could be used like the MPEG-21 RDD, the ODRL vocabulary [4] or the Access Management Ontology [5]. On the other hand, the Creative Commons licensing framework [6] allows users to share content as they like but with some possible restrictions on its reuse (no commercial use, attribution, no derivative, etc.). The IPR management systems can be classified in Digital Rights Management (DRM) systems allowing to control 'all' the possible different uses of a digital content (e.g. distribution, enhancement, adaptation) and Conditional Access Systems (CAS) allowing to control only the access to the digital resource that is provided with some encryption. Currently the digital industry is much more focused on CAS and some solutions are present mainly for audio/video content access developed by Microsoft, Apple and Adobe for
PCs/mobile/tablets and by Irdeto, Nagravision and others for satellite, IPTV, cable TV digital transmissions.

Regarding Performing Arts content of high cultural and economic value, the ECLAP (European Collected Library of Artistic Performance, [7]) ICT PsP project of the European Commission, provides many precious materials, thanks to the Best Practice Network developed. This material is captured on film, video, audio, images, books, posters, etc. As aggregator of Europeana, ECLAP ingests the metadata and digital content. ECLAP has created a considerable online archive for all the performing arts in Europe providing solutions and tools to help performing arts institutions to enter the digital Europe by building a network of important European performing arts institutions and archives and publishing content collections on Europeana, the European Digital Library, [9], [10]. ECLAP is presently distributing more than 100,000 content objects, coming from more than 35 prestigious European institutions (for more details see [11]) images, video, documents, audio, slides, play lists, collections, annotations, etc. In this paper, the IPR Management facilities and tools of ECLAP are presented.

The paper is organized as follows: in section II the ECLAP IPR Management solution is presented; in section III the ECLAP Overall Scenario is described; in section IV the IPR Logic model is detailed; in section V the IPR Wizard tool description is presented. Section VI describes a statistical analysis related to the IPR Wizard tool usage on ECLAP portal and content ingestion and distribution service. Conclusions are drawn in section VII.

\section{ECLAP IPR MANAGEMENT REQUIREMENTS}

In the ECLAP service, Content Providers provide both content files and metadata. According to ECLAP workflow, the obtained metadata sets are sent to Europeana only after that the metadata have been enriched and linked to a reachable digital resource and when the IPR issues have been correctly defined. Moreover, the assignment of access restrictions is a way to enable the increment of possible available content on the internet. Permissions can be applied on content by each ECLAP Content Provider, CP, using an IPR Wizard tool. The process of enriching cross media multilingual metadata related to each content is complex and provide them as public domain (CC0 1.0 Universal Public Domain License) and augmented by technical and multilingual metadata, [13]. In this context, ECLAP does 
not act only as an intermediary between the Content Provider, $\mathrm{CP}$, and Europeana but aggregates and maintains the content files: the links to the contents that ECLAP provides to Europeana and that lead to the real content file. Moreover, ECLAP supports the CPs in all their work: starting from the ingestion, passing through the definition and management of permissions and licenses on contents, managing the real content and providing to all the ECLAP users the typical services of a Social Network. One of the most important aspects and that we will treat in this paper is related to the management of IPR.

The main problems related to the IPR management in ECLAP, as well as in many cultural heritage communities playing the role of content aggregator and content provider service, are the following:

The provided solution has to:

- (CP and Rights) avoid to the Content Partners the assigning of inconsistent rights on objects.

- (IPR Models Definition) allow the CP the possibility of creating their personal IPR models.

- (Application of IPR Models) give the possibility to apply an IPR model to $\mathrm{N}$ digital contents and make sure that this model can be redefined without editing contents again.

- (Association of IPR Models at Ingestion Time) allow to associate the model to the contents (single or entire collections) at the ingestion time, or at any phase of the workflow

- (IPR Model's Additional Conditions) allow to insert in the template some additional conditions according to MPEG-21, ODRL, OASIS XAMCL, such as nationality, times of plays, temporal usage.

In the next sections, each of these issues just outlined, are described in details.

\section{A. Content Providers and Rights}

Avoid that the Content Partners (CPs) can incorrectly assign licenses to the contents is the point from which the work on the IPR management started. In fact the first step made by the ECLAP Consortium was to help the CPs to: (i) understand their rights on digital contents, (ii) guide them on choosing what type of restrictions, if needed, they wanted to impose on their content once having put it online, (iii) inform them about available technologies and on the IPR issues related to them, (iv) avoiding the definition of inconsistent rights on objects (Inconsistency can be due to the definition of limitations that cannot be enforced in a given context. For example, they may request to avoid images to be copied if they are visualized on a computer). As it has happened in other studies or in the development of standards, the relationships among the rights identified have been analysed, formalized and the logic that relates them has been implemented in the ECLAP IPR Wizard which is based on ECLAP IPR model presented in this paper, see user manual [8].

Once the CPs have understood, from a legal point of view, their position with respect to the content and its reuse on the web, the next stage was to guide them on defining licenses using the tools provided by ECLAP in order to formalize the IPR Models with related access restriction/permissions.

\section{B. IPR Models Definition}

Given the diversity of CPs and of the related needs on their contents, a general and flexible IPR model has to be defined. The solution can produce specialized IPR models for each CP. In this way it is possible to customize the binding of licenses and permissions based on the specific needs and with the greatest flexibility. An IPR Model contains:

- model details: IPR Model name, description, etc.;

- a set of permissions such as: play, download, embed, etc. and differently defined for PC (web) and mobile devices; Different permissions for

- different content kind (audio, video, images, document, etc.);

- different resolutions, etc.

- a license (Creative Commons, etc.);

- a Publisher ECLAP page (related to the Content Provider, right owner);

- an IPR ingestion identifier (needed to assign the IPR Model to the contents).

In ECLAP, the users that can create and manage $I P R$ Models are called IPR Managers.

\section{Application of IPR Models}

An IPR Model, once defined, can be associated with a content manually from the interface of the ECLAP workflow or automatically. If a CP has the needs to change the access permissions or licenses associated with content may do so by going directly to change the IPR Model. The association <IPR Model; content> remains unchanged: the permissions on content are instantly updated to all content.

This was performed by giving the possibility to each CP to provide content with initial maximum restrictions: the content, at first, is accessible only for Trusted Users. Moreover, ECLAP gives to the public users the visibility of some metadata (those in public domain) on the regular user. While, public users cannot access the digital content until the content is not associated with an IPR Model, but can see their existence with the possibility of contacting the $\mathrm{CP}$ in case they were interested in the content. By associating an IPR Model to one or more content, each CP can change the initial maximum restriction access allowing external users to possibly access content depending on the conditions expressed in the model.

\section{Association of IPR Models at Ingestion Time}

ECLAP $\mathrm{CP}$ has to manage a huge quantity of digital contents, so the modality (described in section $C$.) of make the association <IPR Model; content> one by one, is not sufficient. For this reason, the presence of an IPR ingestion identifier (IPR_id) in the IPR Model has been provided. This identifier is obviously also connected to the contents as metadata. In this way, a CP can associate an IPR Model 
with contents also in case of massive ingestion and workflow, that in ECLAP is the standard way to upload content. Each CP could create several IPR Models, and may put the corresponding IPR_id as metadata on the content and the system automatically manages the association.

\section{E. IPR Model's Additional Conditions}

Each IPR Model is made in such a way that even the definition of additional conditions is allowed, in line with the standard MPEG-21, ODRL, OASIS XAMCL. Some of these data may be, for example: the expiration date, the duration of the validity, etc.

\section{ECLAP OVERALL SCENARIO}

In order to better understand the IPR management, it is useful to describe the ECLAP Overall Scenario in terms of flow of actions, rules, procedures, etc., that each Content Provider follow to publish content on ECLAP and then provide it to Europeana (Fig. 1).

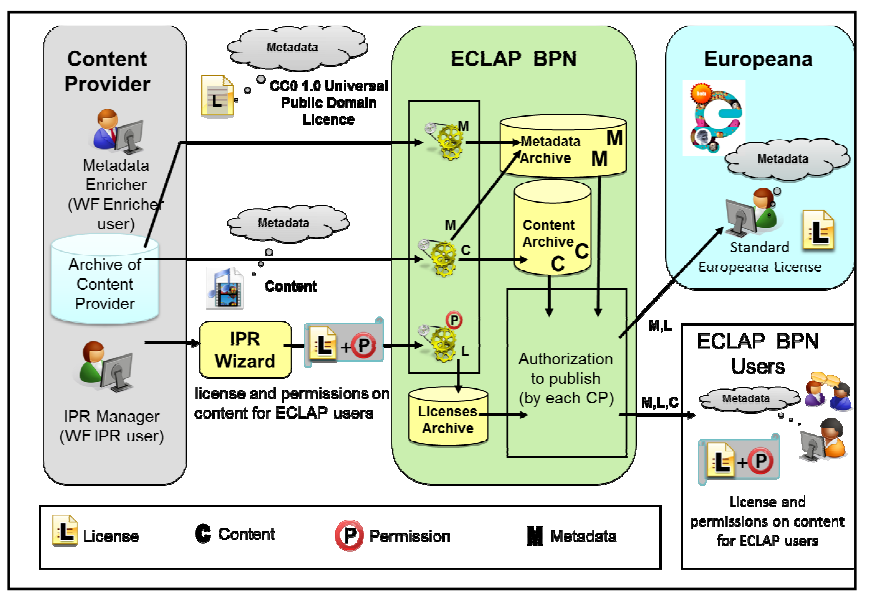

Fig. 1. ECLAP Overall Scenario

All the content managed in the ECLAP must be associated with a specific workflow before it can be connected to Europeana via its metadata. In the cases of Europeana based ECLAP workflow, each content has to be:

- uploaded;

- enriched through metadata (some metadata must be sent to Europeana and others are necessary to describe and manage the content in the ECLAP);

- $\quad$ associated to an IPR Model (through the IPR Wizard, see next sections).

As said before, the content uploaded/ingested is initially available on the ECLAP BPN with maximum restrictions. While metadata are immediately available for indexing and searching for all kind of ECLAP users. Only content presenting a (i) sufficient set of metadata (e.g., Europeana mandatory metadata) and (ii) IPR information and a license defined (one from the set admitted by "europeana:rights"), will be published on Europeana, [12]. In ECLAP, many different set of permissions on the content are available and take into account both ECLAP Content Providers' needs. For example: content and metadata upload methods; metadata standards and formats; IPR on content (licenses, permissions, etc.); collection topics; etc. So for the pdf/images/ animations/html/etc. three permissions are present while for the audio and video permissions and their relations are more articulated Fig. 2. Permissions managed on the ECLAP Portal [8] can be referred to the following aspects:

- access to the content (e.g., the content can be accessible via progressive download and/or download)

- user device (e.g., the content can be played via a PC and/or a mobile device, iPad, etc.)

- content resolution (e.g., the content can be accessible only in a reduced Low Resolution and/or in High resolution).

Moreover, many users with different roles and permissions are involved in the ECLAP knowledge workflow. An important thing to be noticed is the concept of group: in ECLAP each CP has its own group (distribution channel) and can manage only the content uploaded by a user registered to its group. This is a guarantee that the contents uploaded on the portal are only managed by whom has the rights to do so.

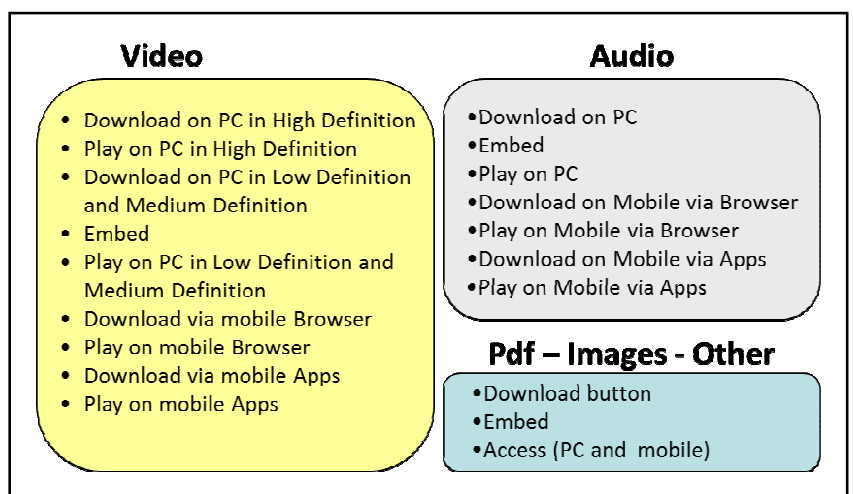

Fig. 2. ECLAP Permissions

\section{IPR MODEL: LOGIC RELATIONSHIPS AMONG RIGHTS}

The IPR Wizard tool has been realized in order to guide the CP (or more precisely, the IPR Managers chosen by each $\mathrm{CP}$ ) on creating an IPR Models and on making the association <IPR Model; content>. This tool is realized starting from the IPR Logic Model that takes into account all the issues related to publishing content online in the ECLAP context, described in synthesis in the previous paragraphs, and is created to simplify and make systematic and standard the work of the IPR Managers of each CP. The IPR Logic Model is based on the following two main aspects, approved by the 35 ECLAP international partners:

- relationships among user roles;

- relationships among permissions. 


\section{A. Relationships among user roles}

The users involved in the IPR management can be users registered to the ECLAP or not (e.g., public users). Each registered user may have additional roles: each role can have a set of IPR permissions associated to it. The IPR Manager can establish the set of permissions for each single digital content through the IPR Wizard. It is possible to establish a hierarchy among the user roles, that are: Public User (PU, not registered), Group User (registered and enrolled to the CP's group), Group and Educational Users (registered, enrolled to CP's group and that has declared in his/her user profile to be an educational/research user), Trusted User (TU, user that belong to ECLAP partners). It should be noted that the IPR Managers are Trusted Users with the specific task on IPR. The hierarchy is explained in Fig. 3, in which the arrows representing the relations on how to assign the permissions to the users. For example: if an IPR Manager assigns a permission to a Public User, the system has to automatically associate the same permissions to all the registered users (Group Users and Group and Educational Users). Note that the Trusted Users always have all the permissions.

\begin{tabular}{|l|c|c|c|c|}
\hline \multirow{2}{*}{ Permissions } & \multirow{2}{*}{ Trusted User } & \multirow{2}{*}{$\begin{array}{c}\text { Public } \\
\text { User }\end{array}$} & \multicolumn{2}{|c|}{ User subscribed to groups } \\
\cline { 4 - 5 } & & & Group User & $\begin{array}{c}\text { Group and } \\
\text { Educational User }\end{array}$ \\
\hline Perm_1 & $\checkmark$ & & & \\
\hline Perm_2 & $\checkmark$ & $\square$ & & \\
\hline Perm_3 & $\checkmark$ & & & \\
\hline
\end{tabular}

Fig. 3. Relationships among user roles.

\section{B. Relationships among permissions}

The relationships among the permissions (or rights) identified have been analysed on the basis of the content type to which they are applied and modelled basing on logical and technical aspects. In Fig. 4, the arrows are posed to explain that some permissions implicitly involves other permissions.

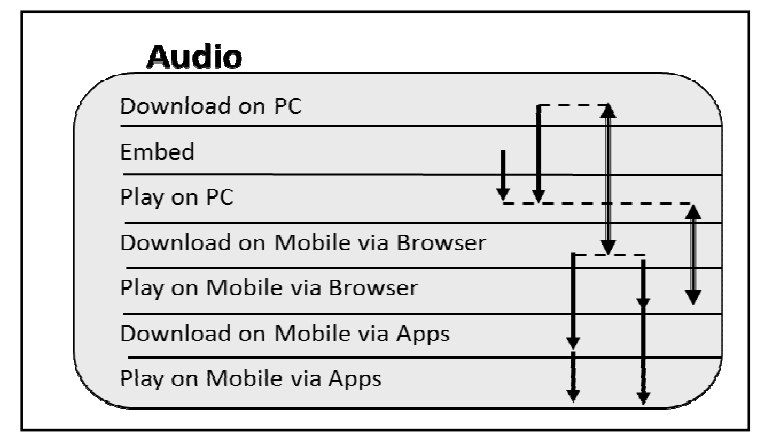

Fig. 4. IPR permissions relations on Audio content

Here after two samples on audio content (same samples can be done also for the pdf/images/other or video content), useful to describe the permission relations. The relations among permissions are represented as different arrows:
Unidirectional arrow between 'Audio download-PC' $\rightarrow$ 'Audio play-PC': if a Content Partner allows an ECLAP user to download the audio content, the CP implicitly allows also to play it (play via streaming and/or progressive download). This because, from a technical point of view: if someone downloads a content (without encryptions or protection) from the web he can play/view it on his PC whenever he wants.

Bidirectional arrow between 'Audio download-PC' $\longleftrightarrow$ 'Audio download-mobile-Browser': if a Content Partner allows an ECLAP user to download a content from PC, implicitly allows him also to download the same content from a mobile device. This because the users can download a content via a browser in their PC, then transfer the content into a mobile device, so that the application of a restriction to avoid the download via mobile can be easily moved around and has no sense to be applied. It is also true the vice-versa.

\section{IPR WIZARD TOOL}

The IPR Wizard creates a new IPR Model starting with: "All permissions for TU and no permissions for the other users" as default values. The IPR Logic Model implemented takes decisions for the IPR Managers according to the hierarchy of relationships (see previous sections). The manager has just to select one or more permissions that he/she wants to associate to an IPR Model (and therefore to a set of contents) and the wizard automatically selects also the permissions strictly connected to those chosen by the IPR Manager. This mechanism has two main advantages: the IPR Manager does not need to know the relationships among the permissions; the probability of error for inconsistency is null.

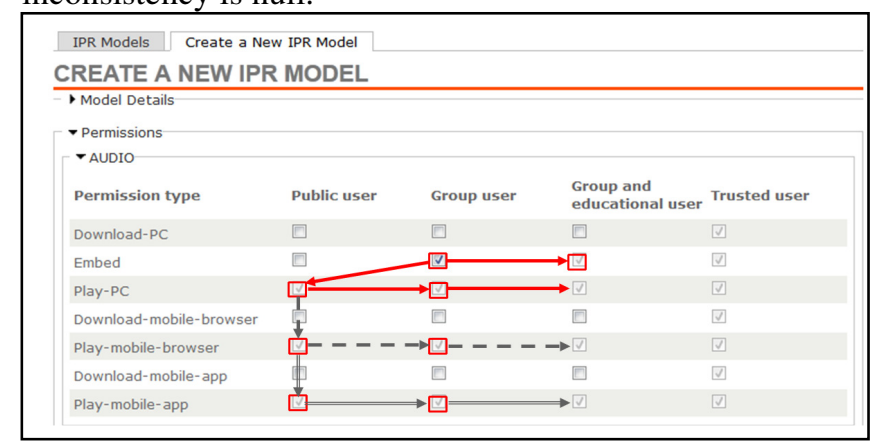

Fig. 5. IPR Wizard: audio sample.

A sample, in Fig. 5: "If a CP allows all Group Users to embed an audio content", the IPR Wizard directly implies the following permissions on audio content:

- $\quad$ Step 1 ('Embed' $\rightarrow$ 'Play-PC'; relationships among users): (i) all the users (Public, Group, Group and Educational) can play the content on PC; (ii) Group and Educational Users can embed the content;

- Step 2 ('Play-PC' $\rightarrow$ 'Play-mobile-browser'; relationships among users): (i) all the users (Public, 
Group, Group and Educational) can play the content on mobile via Browser;

- Step 3 ('Play-Mobile-Browser' $\rightarrow$ 'Play-mobileapp'; relationships among users): (i) all the users (Public, Group, Group and Educational) can play the content on mobile via ECLAP Applications.

In this case not all permissions to all users are allowed: the Creative Commons Licences cannot be associated with this IPR Model, so the user can choose the licence from one of the restricted licences allowed by Europeana ("Unknown copyright status" or "Right Reserved - Restricted access"), [12]. While if a CP creates an IPR Model in which all the permissions are allowed to all the users, it is possible to choose one of the CC Licences.

\section{IPR WIZARD TOOL USAGE}

ECLAP IPR Wizard is largely used by more than 35 partners in Europe. As of January 2013 there are in use 49 IPR models, 30 are restrictive not public models while 19 are public models. Most CPs used 1, 2 or 3 models for their content but there are three partners that used even 4,8 or 12 models. Fig. 6 reports for each model how many content are associated with it, it can be seen that two models cover more than the half of the content, but also the high flexibility.

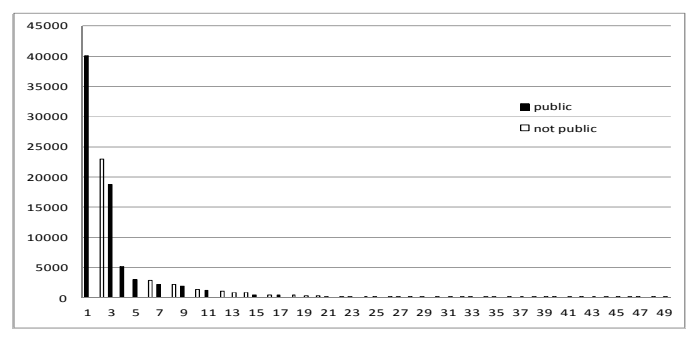

Fig. 6. Statistics on IPR Models.

The $68 \%$ of the content is associated with a public IPR model. Regarding the 30 restrictive IPR models defined by CPs, in 11 cases they restricted the access to only educational group users and in 6 cases restricted the access to group users only (educational and not educational). Moreover, 23 models are used to not allow the download of the digital resource for some kind of resource type (regardless of the user type).

TABLE I. IPR MODELS ALLOWING PERMISSIONS BY USER TYPE

\begin{tabular}{|l|l|c|c|}
\hline \multirow{2}{*}{\multicolumn{1}{|c|}{ Permission }} & \multicolumn{3}{c|}{ User type } \\
\cline { 2 - 4 } & public & group & educ./research \\
\hline only play/access & 11 & 13 & 19 \\
\hline download \& play & 3 & 8 & 11 \\
\hline no permission & 19 & 12 & 4 \\
\hline
\end{tabular}

Table I reports, for the three user types (public, group and educational), how many IPR models allow only play/access of the digital resource or allow the download and play of it or no permission are provided. It can be seen that in most cases the models are used to restrict access from the public users (19 over 30) and to limit the download of the resource.

\section{CONCLUSIONS AND FUTURE WORK}

ECLAP portal is widely accessed from the world, with 1500 experts users. ECLAP IPR Wizard is largely used by more than 35 content providers in Europe. The IPR model and wizard proposed covers the needs of content providers and it is an innovative solution with respect of the state of the art. For the future main improvements would be to (i) define an IPR Model Wizard for federated social media content and services, (ii) increase the number of rights addressed and controlled such as: aggregation, composition, excerpts, etc. (iii) increase the number of conditions addressed and controlled such as: localization, user certificates, languages, ages, etc. (iv) formalize the solution as a standard and propose to some standard bodies as MPEG, W3C, etc. (v) developing an IPR wizard and player enforcing as federated service; validate and test it.

\section{ACKNOWLEDGMENT}

The authors want to thank all the partners involved in ECLAP, and the European Commission for funding ECLAP in the Theme CIP-ICT-PSP.2009.2.2, Grant Agreement No. 250481.

\section{REFERENCES}

[1] X. Wang, "MPEG-21 rights expression language: Enabling interoperable digital rights management," IEEE Multimedia, 11(4):84-87, 2004.

[2] R. Iannella, S. Guth, D. Pähler, and Andreas Kasten. ODRL version 2.0 core model. Specification, W3C ODRL Community Group, 04 2012. http://www.w3.org/community/odrl/two/model/

[3] T. Moses, "Privacy policy profile of XACML v2.0," Oasis standard, OASIS, 02 2005. http://docs.oasis-open.org/xacml/2.0/PRIVACYPROFILE/access_control-xacml-2.0-privacy_profile-spec-os.pdf

[4] R. Iannella and S. Guth. ODRL version 2.0 common vocabulary. Specification, W3C ODRL Community Group, 042012. http://www.w3.org/community/odrl/two/vocab/

[5] M. Buffa and C. Faron-Zucker. Ontology-based access rights management, "In Advances in Knowledge Discovery and Management," vol. 398, Studies in Computational Intelligence, pp 49-61. Springer, 2012.

[6] Creative Commons, http://creativecommons.org

[7] European Library of Artistic Performance, ECLAP, http://www.eclap.eu/

[8] "ECLAP DE3.1 infrastructure: ingestion and processing content and metadata," 2011, ECLAP Project, http://www.eclap.eu/ urn:axmedis:00000:obj:a345a84f-6fdf-4f84-a412-88094ce363e2

[9] Europeana, http://www.europeana.eu

[10] M. Paolucci, "Models and Mechanisms for Social and Best Practice Networtks", Ph.D. program in "Telematica e Società dell'Informazione", Cycle XIV.

[11] ECLAP Partners. List and information on ECLAP Partners available at: http://www.eclap.eu/drupal/?q=node/3578

[12] 'Guidelines for the europeana:rights metadata element', v4.0 - 20

[13] P. Bellini, D. Cenni, P. Nesi, "On the Effectiveness and Optimization of Information Retrieval for Cross Media Content", Proc. of the KDIR 2012, Part of IC3K 2012, Int. Joint Conf. on Knowledge Discovery, Knowledge Engineering and Knowledge Management. Barcelona, Spain, 4-7 October 2012. 